\section{Réduire l'immunosuppression par les Treg avec des anticorps monoclonaux anti-GARP}

\author{
Sophie Lucas
}

La seconde provient de l'existence, chez la souris en tout cas, de divers mécanismes d'immunosuppression par les Treg, comme la production de cytokines immunosuppressives, la diminution de la capacité des cellules présentatrices d'antigènes à stimuler les lymphocytes $T$, le transfert d'AMP cyclique à travers les jonctions communicantes ${ }^{2}$, ou encore la production d'adénosine. L'importance d'un mécanisme particulier semble dépendre du contexte et du type de réponse immunitaire à supprimer. Lequel de ces mécanismes, ou d'autres encore non identifiés, joue un rôle prépondérant chez l'homme n'est pas connu.

Pour étudier les mécanismes d'immunosuppression par les Treg humains, nous avons dérivé des clones de Treg, afin de contourner la difficulté inhérente à l'isolement répété de ces cellules sanguines rares, difficilement identifiables, et présentant une fonction suppressive peu reproductible in vitro. Un clone est défini comme Treg s'il possède une marque épigénétique stable, qui ne s'établit que dans les Treg complètement différenciés chez l'homme et chez la souris. Cette marque correspond à la déméthylation d'une région régulatrice du gène FOXP3 $[1,2]$. Nos clones Treg expriment FOXP3, sont suppresseurs in vitro, et se sont révélés une source de cellules remarquablement stables et pures pour étudier la fonction immunosuppressive des Treg humains. L'analyse

\footnotetext{
${ }^{2}$ Les jonctions communicantes (ou gap junctions en anglais) sont des structures membranaires permettant la diffusion intercellulaire de petites molécules.
}

Institut de Duve, avenue Hippocrate, 74, 1200 Bruxelles, Belgique.

sophie.lucas@uclouvain.be

de leur profil transcriptionnel a montré qu'en réponse à une stimulation du récepteur $\mathrm{T}$, les Treg humains, mais pas les autres types de lymphocytes $T$, produisent la forme active du TGF- $\beta$ l (transforming growth factor- $\beta 1$ ) [2]. Les souris $\mathrm{Tgfbl}^{-/-}$souffrent d'un syndrome auto-immunitaire létal. II semblait donc raisonnable de postuler que la production de cette cytokine par les Treg contribue à leur fonction suppressive. Cependant, la suppression par les Treg requiert des contacts cellulaires, ce qui suggère plutôt un mécanisme impliquant des protéines membranaires. Mais nous avons ensuite observé que l'induction d'un signal dépendant du TGF- $\beta 1$ dans des lymphocytes $T$ en présence de Treg requérait également des contacts cellulaires, indiquant que le TGF- $\beta$ l actif est produit à proximité de la surface des Treg [2]. Ces observations nous ont conduits à examiner en détails les mécanismes d'activation du TGF- $\beta 1$ par les Treg humains. En effet, la plupart des cellules produisent des formes inactives du TGF- $\beta 1$ mais seul un petit nombre sont capables d'activer la cytokine, via des mécanismes très contrôlés qui varient d'un type cellulaire à l'autre [3]. Toutes les cellules immunitaires produisent le précurseur du TGF- $\beta$ l, le proTGF- $\beta l$ qui est clivé pour générer le TGF- $\beta$ l latent dont le fragment carboxy-terminal, correspondant au TGF- $\beta 1$ mature, reste lié de manière non covalente au fragment amino-terminal appelé LAP (latency associated peptide). Le TGF- $\beta$ l latent est inactif parce que le LAP empêche la liaison du TGF- $\beta 1$ mature à son récepteur 


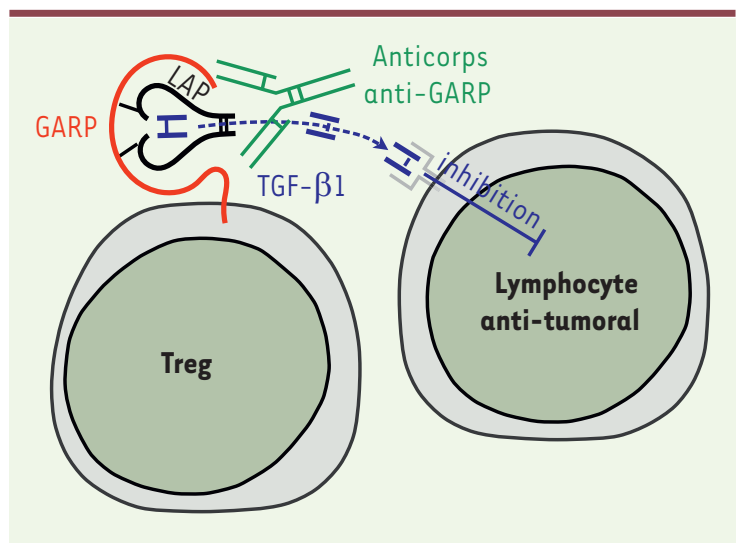

Figure 1. Mode d'action des anticorps anti-GARP. Le TGF$\beta l$ actif libéré des complexes GARP/TGF- $\beta 1$ à la surface des Treg inhibe les lymphocytes $T$ à proximité. Des anticorps anti-GARP peuvent bloquer la production de TGF- $\beta 1$ actif par les Treg, et donc réduire l'activité immunosuppressive de ces derniers in vivo.

(Figure 1). Une étape supplémentaire est en effet nécessaire pour libérer le TGF- $\beta 1$ mature du LAP. Avec d'autres équipes, nous avons montré que les Treg stimulés, mais pas les autres types de lymphocytes $T$, présentent le TGF- $\beta 1$ latent à leur surface grâce à sa liaison à une protéine transmembranaire appelée GARP (glycoprotein A repetitions predominant) $[4,5]$. Nous avons postulé, et ensuite démontré, que GARP contribuait à la production de TGF- $\beta 1$ actif par les Treg. Nous avons obtenu 31 nouveaux anticorps anti-GARP, dont 2 se sont avérés capables d'inhiber la production de TGF- $\beta 1$ actif par les Treg humains. Ces deux anticorps reconnaissent un épitope conformationnel qui requiert les acides aminés situés en position 137-139 au sein des complexes GARP/ TGF- $\beta 1$ (GARP $137-139)$. Les autres anticorps, qui ne bloquent pas l'activation du TGF- $\beta 1$, reconnaissent d'autres épitopes. Nous avons mesuré l'activité de ces anticorps anti-GARP bloquants chez des souris immunodéficientes, greffées avec des PBMC (cellules mononucléées isolées du sang périphérique humain). Ces souris développent une maladie du greffon contre l'hôte (en anglais, graft-versus-host disease, GVHD) provoquée par l'activité des lymphocytes $T$ humains greffés contre les tissus de la souris receveuse. Le co-transfert de Treg autologues ${ }^{3}$ humains permet d'atténuer

\footnotetext{
${ }^{3}$ Autologue caractérise un tissu ou des cellules provenant de son propre organisme et autoadministré.
}

la GVHD, et, inversement, les anticorps anti-GARP bloquants abolissent cette protection. Les anticorps n'agissent pas en éliminant les Treg : le nombre de Treg humains n'est en effet pas diminué dans les souris traitées avec les anticorps anti-GARP bloquants. De plus, un anticorps présentant une mutation qui abolit sa capacité de se lier aux récepteurs $\mathrm{Fc}$, conserve une activité totale. Nos résultats indiquent donc: (i) que la production de TGF- $\beta 1$ actif via GARP contribue à la fonction suppressive des Treg humains in vivo et (ii) que des anticorps anti-GARP peuvent inhiber cette immunosuppression in vivo, sans éliminer les Treg [6].

La notion que le TGF- $\beta 1$ contribue, ne serait-ce que partiellement, à l'immunosuppression exercée par les Treg, est loin d'être acceptée, notamment parce que les Treg de souris $\operatorname{Tgfbl}^{-/-}$ ne montrent pas d'anomalie de leur fonction suppressive in vitro [7]. Plusieurs études suggèrent néanmoins que le TGF- $\beta$ l produit par les Treg supprime l'autoimmunité in vivo [8, 9]. II est possible d'autre part que les Treg humains puissent supprimer les réponses immunes via des mécanismes qui seraient distincts de ceux utilisés par les Treg murins.

Quels avantages les anticorps anti-GARP pourraient-ils apporter à l'immunothérapie du cancer?

D'une part, il convient de noter qu'aucun des anticorps immunostimulants actuellement disponibles en clinique, n'agit en inhibant la fonction immunosuppressive des Treg. Les anticorps antiCTLA-4 pourraient intervenir en partie en éliminant les Treg dans les tumeurs. Ce mode d'action a été démontré chez la souris [10], et pourrait aussi jouer un rôle chez les patients chez lesquels les anticorps anti-CTLA-4 provoquent des effets secondaires sévères de nature auto-immunitaire. II est donc tentant de spéculer que l'administration d'anticorps anti-GARP, qui inhibent transitoirement la fonction des Treg sans les éliminer physiquement, serait moins toxique que l'administration d'anticorps anti-CTLA-4.

D'autre part, les anticorps anti-GARP ne devraient pas affecter la production de TGF- $\beta 1$ actif par les cellules autres que des Treg. Ceci pourrait être avantageux par rapport à une inhibition globale qui serait obtenue avec des anticorps anti-TGF- $\beta 1$, ou des inhibiteurs de l'activité kinase des récepteurs du TGF- $\beta$. Une telle inhibition de l'activité du TGF- $\beta$ l, qui est produit par tous les types cellulaires, pose le problème du risque d'effets secondaires sévères, comme la stimulation de la croissance de lésions pré-néoplasiques, puisque le TGF$\beta 1$ exerce de puissants effets cytostatiques sur les cellules précancéreuses. Les anticorps anti-GARP devraient permettre une inhibition sélective de l'activité du TGF- $\beta 1$ dans les cellules immunitaires supprimées par les Treg. $\diamond$

Immunosuppression by Treg can be decreased with anti-GARP antibodies

\section{LIENS D'INTÉRÊT}

Les auteurs déclarent n'avoir aucun lien d'intérêt concernant les données publiées dans cet article.

\section{RÉFÉRENCES}

1. Josefowicz SZ, Lu LF, Rudensky AY. Regulatory T cells: mechanisms of differentiation and function. Annu Rev Immunol 2012 ; 30 : 531-64.

2. Stockis J, Fink W, Francois V, et al. Comparison of stable human Treg and Th clones by transcriptional profiling. Eur J Immunol $2009 ; 39: 869-82$.

3. Travis MA, Sheppard D. TGF-beta activation and function in immunity. Annu Rev Immunol 2014 ; 32 : 51-82.

4. Stockis J, Colau D, Coulie PG, Lucas S. Membrane protein GARP is a receptor for latent TGF-beta on the surface of activated human Treg. Eur J Immunol 2009; 39 : 3315-22. 


\section{RéFÉRENCES}

5. Tran DQ, Andersson J, Wang R, et al. GARP (LRRC32) is essential for the surface expression of latent TGF-beta on platelets and activated FOXP3+ regulatory T cells. Proc Natl Acad Sci USA 2009 ; 106 : 13445-50.

6. Cuende J, Liénart S, Dedobbeleer 0 , et al. Monoclonal antibodies against GARP/TGF- $\beta 1$ complexes inhibit the immunosuppressive activity of human regulatory T cells in vivo. Sci Transl Med $2015 ; 7$ : 284ra56.
7. Piccirillo CA, Letterio JJ, Thornton AM, et al. CD4(+)CD25(+) regulatory $T$ cells can mediate suppressor function in the absence of transforming growth factor betal production and responsiveness.J Exp Med 2002 ; 196 : 237-46.

8. Marie JC, Liggitt D, Rudensky AY. Cellular mechanisms of fatal early-onset autoimmunity in mice with the $T$ cell-specific targeting of transforming growth factorbeta receptor. Immunity 2006 ; 25 : 441-54.

9. Li MO, Wan Yy, Flavell RA. T cell-produced transforming growth factor-betal controls $T$ cell tolerance and regulates Thl- and Th17-cell differentiation. Immunity $2007 ; 26: 579-91$.
10. Simpson TR, Li F, Montalvo-Ortiz W, et al. Fc-dependent depletion of tumor-infiltrating regulatory $T$ cells co-defines the efficacy of antiCTLA-4 therapy against melanoma. J Exp Med 2013 ; $210: 1695-710$.

11. Siri A, de Boysson H, Boursier G. Actualité sur les lymphocytes T régulateurs CD4 ${ }^{+}$. Med Sci (Paris) 2012 ; $28: 646-51$.

\section{NOUVELle}

\section{Trafic cellulaire des récepteurs $\mathrm{GABA}_{B}$ vers la membrane: PRAF2, un nouveau point de contrôle}

Stéphane Doly, Stefano Marullo
Institut Cochin, Inserm U1016, CNRS UMR8104, université Paris Descartes, 27 rue du faubourg Saint-Jacques, 75014 Paris, France.

stefano.marullo@inserm.fr
> Les récepteurs couplés aux protéines $G$ (RCPG), dont on compte plusieurs centaines de membres, représentent la plus grande famille de récepteurs membranaires ${ }^{1}$ [17]. Ils sont impliqués dans de nombreux processus pathologiques et représentent actuellement environ $30 \%$ des cibles thérapeutiques médicamenteuses.

Les signaux résultant de l'activation des $R C P G$, et par conséquent leurs effets physiologiques, dépendent de leur densité à la surface cellulaire et de leur adressage dans des régions spécifiques de la membrane plasmique telles que les synapses pour les cellules neuronales. La plupart des données actuelles sur le trafic cellulaire des RCPG concernent leur internalisation (par endocytose) après qu'ils aient été activés par leurs ligands agonistes, leur recyclage ou leur orientation vers la voie de dégradation lysosomiale. En revanche, les mécanismes qui régulent l'export de ces récepteurs des compartiments de biosynthèse vers la surface cellulaire, sont beaucoup moins bien connus. En effet, il est généralement admis que les RCPG nouvellement synthétisés sont localisés dans des vésicules qui fusionnent avec la membrane plasmique sans contrôle

${ }^{1}$ Voir le numéro thématique consacré à ces récepteurs RCPG, $\mathrm{m} / \mathrm{s} \mathrm{n}^{\circ} 10$, vol. 28 , octobre 2012. particulier (voie de sécrétion non régulée). Cependant, de nombreuses observations indiquent que le trafic de ces récepteurs vers la surface cellulaire pourrait être régulé par des interactions avec certaines protéines et par des signaux extra-cellulaires. Des études récentes démontrent que pour de nombreux RCPG, une proportion importante des récepteurs est retenue dans des compartiments intracellulaires comme le réticulum endoplasmique (RE) ou l'appareil de Golgi [1]. Ces « réserves » peuvent être mobilisables vers la membrane plasmique en réponse à une stimulation physiologique qui nécessite une augmentation rapide de la réceptivité cellulaire $[2,3]$. De plus, dans des expériences d'expression des RCPG dans des cellules modèles ne les exprimant pas naturellement (expression hétérologue), il a été montré que beaucoup d'entre eux nécessitent une association à des protéines d'escorte ou à des chaperons moléculaires pour être correctement exprimés à la surface. Les récepteurs olfactifs en sont un des exemples les plus connus et cette caractéristique a pendant longtemps empêché leur étude fonctionnelle in vitro [1]. Dans plusieurs pathologies humaines, comme le diabète insipide néphrogénique, la rétinite pigmentaire, l'obésité ou l'hypogonadisme, des mutations somatiques des RCPG [4] ou de protéines nécessaires à leur maturation [5], sont à l'origine d'un excès de rétention dans les compartiments intracellulaires. Ce défaut s'accompagne d'une altération de la réceptivité à la surface des cellules qui est directement responsable des effets pathologiques observés. Ces récepteurs séquestrés sont cependant potentiellement fonctionnels. Leur capacité de signalisation peut en effet être récupérée sous l'action de chaperons pharmacologiques, capables de franchir les membranes cellulaires et de se lier à eux pour favoriser leur adressage en surface $[6,7]$.

Un des RCPG les plus étudiés comme modèle de rétention intracellulaire est la sous-unité $\mathrm{GBl}$ du récepteur métabotropique $G A B A_{B}$. Le GABA (acide gamma-amino butyrique) est le principal neuromédiateur inhibiteur du cerveau. Il contrôle de nombreuses fonctions sensorimotrices et cognitives. Les récepteurs $G_{A B A_{B}}$ sont des hétérodimères obligatoires composés de deux sous unités $G B 1$ et $G B 2$. Dans l'hétérodimère, $\mathrm{GBl}$ lie le $\mathrm{GABA}$, tandis que GB2 est responsable de l'activation de la protéine $G$; en absence de formation de l'hétérodimère, il ne peut donc pas y avoir de récepteurs $G A B A_{B}$ fonctionnels. $L a$ sous-unité $G B 1$, exprimée seule, est retenue de manière constitutive dans le réticulum endoplasmique. Elle n'est libérée puis adres- 